\title{
Self-Consistent Equations Including Exchange and Correlation Effects*
}

\author{
W. KoHn and L. J. Sham \\ University of California, San Diego, La Jolla, California
}

(Received 21 June 1965)

\begin{abstract}
From a theory of Hohenberg and Kohn, approximation methods for treating an inhomogeneous system of interacting electrons are developed. These methods are exact for systems of slowly varying or high density. For the ground state, they lead to self-consistent equations analogous to the Hartree and Hartree-Fock equations, respectively. In these equations the exchange and correlation portions of the chemical potential of a uniform electron gas appear as additional effective potentials. (The exchange portion of our effective potential differs from that due to Slater by a factor of $\frac{2}{3}$.) Electronic systems at finite temperatures and in magnetic fields are also treated by similar methods. An appendix deals with a further correction for systems with short-wavelength density oscillations.
\end{abstract}

\section{INTRODUCTION}

$I^{\mathrm{N}}$ $N$ recent years a great deal of attention has been given to the problem of a homogeneous gas of interacting electrons and its properties have been established with a considerable degree of confidence over a wide range of densities. Of course, such a homogeneous gas represents only a mathematical model, since in all real systems (atoms, molecules, solids, etc.) the electronic density is nonuniform.

It is then a matter of interest to see how properties of the homogeneous gas can be utilized in theoretical studies of inhomogeneous systems. The well-known methods of Thomas-Fermi ${ }^{1}$ and the Slater ${ }^{2}$ exchange hole are in this spirit. In the present paper we use the formalism of Hohenberg and $\mathrm{Kohn}^{3}$ to carry this approach further and we obtain a set of self-consistent equations which include, in an approximate way, exchange and correlation effects. They require only a knowledge of the true chemical potential, $\mu_{h}(n)$, of a homogeneous interacting electron gas as a function of the density $n$.

We derive two alternative sets of equations [Eqs. (2.8) and (2.22)] which are analogous, respectively, to the conventional Hartree and Hartree-Fock equations, and, although they also include correlation effects, they are no more difficult to solve.

The local effective potentials in these equations are unique in a sense which is described in Sec. II. In particular, we find that the Slater exchange-hole potential, besides its omission of correlation effects, is too large by a factor of $\frac{3}{2}$.

Apart from work on the correlation energy of the homogeneous electron gas, most theoretical many-body studies have been concerned with elementary excitations and as a result there has been little recent progress in the theory of cohesive energies, elastic constants, etc., of real (i.e., inhomogeneous) metals and alloys. The methods proposed here offer the hope of new progress in this latter area.

* Supported in part by the U. S. Office of Naval Research.

${ }^{1}$ L. H. Thomas, Proc. Cambridge Phil. Soc. 23, 542 (1927); E. Fermi, Z. Physik 48, 73 (1928).

2 J. C. Slater, Phys. Rev. 81, 385 (1951).

3 P. Hohenberg and W. Kohn, Phys. Rev. 136, B864 (1964); referred to hereafter as $\mathrm{HK}$.
In Secs. III and IV, we describe the necessary modifications to deal with the finite-temperature properties and with the spin paramagnetism of an inhomogeneous electron gas.

Of course, the simple methods which are here proposed in general involve errors. These are of two general origins $^{4}$ : a too rapid variation of density and, for finite systems, boundary effects. Refinements aimed at reducing the first type of error are briefly discussed in Appendix II.

\section{THE GROUND STATE}

\section{A. Local Effective Potential}

It has been shown ${ }^{3}$ that the ground-state energy of an interacting inhomogeneous electron gas in a static potential $v(\mathbf{r})$ can be written in the form

$E=\int v(\mathbf{r}) n(\mathbf{r}) d \mathbf{r}+\frac{1}{2} \iint \frac{n(\mathbf{r}) n\left(\mathbf{r}^{\prime}\right)}{\left|\mathbf{r}-\mathbf{r}^{\prime}\right|} d \mathbf{r} d \mathbf{r}^{\prime}+G[n]$,

where $n(\mathbf{r})$ is the density and $G[n]$ is a universal functional of the density. This expression, furthermore, is a minimum for the correct density function $n(\mathbf{r})$. In this section we propose first an approximation for $G[n]$, which leads to a scheme analogous to Hartree's method but contains the major part of the effects of exchange and correlation.

We first write

$$
G[n] \equiv T s[n]+E_{\mathbf{x c}}[n],
$$

where $T_{s}[n]$ is the kinetic energy of a system of noninteracting electrons with density $n(\mathbf{r})$ and $E_{\mathbf{x x}}[n]$ is, by our definition, the exchange and correlation energy of an interacting system with density $n(\mathbf{r})$. For an arbitrary $n(\mathbf{r})$, of course, one can give no simple exact expression for $E_{\mathrm{xc}}[n]$. However, if $n(\mathbf{r})$ is sufficiently slowly varying, one can show ${ }^{3}$ that

$$
E_{\mathbf{x c}}[n]=\int n(\mathbf{r}) \epsilon_{\mathbf{x c}}(n(\mathbf{r})) d \mathbf{r},
$$

${ }^{4}$ W. Kohn and L. J. Sham, Phys. Rev. 137, A1697 (1965).

${ }^{5}$ For such a system it follows from HK that the kinetic energy is in fact a unique functional of the density. 
where $\epsilon_{\mathrm{xc}}(n)$ is the exchange and correlation energy per electron of a uniform electron gas of density $n$. Our sole approximation consists of assuming that (2.3) constitutes an adequate representation of exchange and correlation effects in the systems under consideration. We shall regard $\epsilon_{\mathrm{xc}}$ as known from theories of the homogeneous electron gas. ${ }^{6}$

From the stationary property of Eq. (2.1) we now obtain, subject to the condition

the equation

$$
\int \delta n(\mathbf{r}) d \mathbf{r}=0
$$

$$
\int \delta n(\mathbf{r})\left\{\varphi(\mathbf{r})+\frac{\delta T_{\delta}[n]}{\delta n(\mathbf{r})}+\mu_{\mathrm{xc}}(n(\mathbf{r}))\right\} d \mathbf{r}=0
$$

here

$$
\varphi(\mathbf{r})=v(\mathbf{r})+\int \frac{n\left(\mathbf{r}^{\prime}\right)}{\left|\mathbf{r}-\mathbf{r}^{\prime}\right|} d \mathbf{r}^{\prime},
$$

and

$$
\mu_{\mathrm{xc}}(n)=d\left(n \epsilon_{\mathrm{xc}}(n)\right) / d n
$$

is the exchange and correlation contribution to the chemical potential of a uniform gas of density $n$.

Equations (2.4) and (2.5) are precisely the same as one obtains from the theory of Ref. 3 when applied to a system of noninteracting electrons, moving in the given potential $\varphi(\mathbf{r})+\mu_{\mathrm{xe}}(n(\mathbf{r}))$. Therefore, for given $\varphi$ and $\mu$, one obtains the $n(\mathbf{r})$ which satisfies these equations simply by solving the one-particle Schrödinger equation

$$
\left\{-\frac{1}{2} \nabla^{2}+\left[\varphi(\mathbf{r})+\mu_{\mathrm{xc}}(n(\mathbf{r}))\right]\right\} \psi_{i}(\mathbf{r})=\epsilon_{i} \psi_{i}(\mathbf{r}),
$$

and setting

$$
n(\mathbf{r})=\sum_{i=1}^{N}\left|\psi_{i}(\mathbf{r})\right|^{2}
$$

where $N$ is the number of electrons.

It is physically very satisfactory that $\mu_{\mathbf{x c}}$ appears in Eq. (2.8) as an additional effective potential so that gradients of $\mu_{\mathrm{xc}}$ lead to forces on the electron fluid in a manner familiar from thermodynamics.

Equations (2.6)-(2.9) have to be solved self-consistently: One begins with an assumed $n(\mathbf{r})$, constructs $\varphi(\mathbf{r})$ from (2.6) and $\mu_{\mathbf{x c}}$ from (2.7), and finds a new $n(\mathbf{r})$ from (2.8) and (2.9). The energy is given by

$$
\begin{aligned}
E=\sum_{1}^{N} \epsilon_{i}-\frac{1}{2} & \iint \frac{n(\mathbf{r}) n\left(\mathbf{r}^{\prime}\right)}{\left|\mathbf{r}-\mathbf{r}^{\prime}\right|} d \mathbf{r} d \mathbf{r}^{\prime} \\
& +\int n(\mathbf{r})\left[\epsilon_{\mathrm{xc}}(n(\mathbf{r}))-\mu_{\mathrm{xc}}(n(\mathbf{r}))\right] d \mathbf{r} .
\end{aligned}
$$

The results of our procedure are exact in two limiting cases:

(a) Slowely varying density. This regime is characterized by the condition $r_{s} / r_{0} \ll 1$, where $r_{s}$ is the Wigner-

${ }^{6}$ For a review see D. Pines, Elementary Excitations in Solids (W. A. Benjamin, Inc., New York, 1963).
Seitz radius and $r_{0}$ is a typical length over which there is an appreciable change in density. In this case, as shown in $\mathrm{HK}$, we can expand the true exchange and correlation energy as follows:

$$
\begin{array}{rl}
E_{\mathrm{xc}}[n]=\int \epsilon_{\mathrm{xc}}(n) n & d \mathbf{r} \\
& +\int \epsilon_{\mathrm{xc}}{ }^{(2)}(n)|\nabla n|^{2} d \mathbf{r}+\cdots,
\end{array}
$$

where $\epsilon_{\mathbf{x c}}{ }^{(2)}$ is the exchange and correlation portion of the second term in the energy expansion in powers of the gradient operator. In this regime we may similarly expand $T_{s}[n]$ in the form

$$
\begin{array}{rl}
T_{s}[n]=\int \frac{3}{10}\left(3 \pi^{2} n\right)^{2 / 3} & n d \mathbf{r} \\
& +\int t^{(2)}(n)|\nabla n|^{2} d \mathbf{r}+\cdots .
\end{array}
$$

From HK, expecially Sec. III 2, we have the following expression for the energy:

$$
\begin{aligned}
E_{v}[n]= & \int v(\mathbf{r}) n(\mathbf{r}) d \mathbf{r}+\frac{1}{2} \int \frac{n(\mathbf{r}) n\left(\mathbf{r}^{\prime}\right)}{\left|\mathbf{r}-\mathbf{r}^{\prime}\right|} d \mathbf{r} d \mathbf{r}^{\prime} \\
& +\int g_{0}(n) d \mathbf{r}+\int g_{2}{ }^{(2)}(n)|\nabla n|^{2} d \mathbf{r}+\cdots
\end{aligned}
$$

where

$$
\begin{array}{ll}
\text { and } & g_{0}(n)=\left\{\frac{3}{10}\left(3 \pi^{2} n\right)^{2 / 3}+\epsilon_{\mathrm{xc}}(n)\right\} n, \\
& g_{2}{ }^{(2)}(n)=\left\{\epsilon_{\mathbf{x c}}{ }^{(2)}(n)+t^{(2)}(n)\right\} n .
\end{array}
$$

Since in our approximation (2.3), the $|\nabla|^{2}$ term of Eq. (2.11) is neglected, it is clear that for a gas of slowly varying density our expression (2.10) for the energy has errors of the order $|\nabla|^{2}$, or equivalently, of the order $r_{0}^{-2}$.

Surprisingly, our procedure determines the density with greater accuracy, the errors being of order $|\nabla|^{4}$. This is shown in Appendix I.

At this point a comparison of our procedure and that of Slater ${ }^{2}$ may be appropriate. For one thing, Slater's original work does not include correlation effects. ${ }^{7}$ But even the exchange correction is different from ours. To obtain Slater's exchange correction, one may begin by writing the Hartree-Fock exchange operator in the form of an equivalent potential acting on the $k$ th wave function

$$
v_{\mathbf{x} k}(\mathbf{r})=-\sum_{k^{\prime}=1}^{N} \int \frac{\psi_{k}^{*}(\mathbf{r}) \psi_{k^{\prime}} *\left(\mathbf{r}^{\prime}\right) \psi_{k^{\prime}}(\mathbf{r}) \psi_{k}\left(\mathbf{r}^{\prime}\right)}{\left|\mathbf{r}-\mathbf{r}^{\prime}\right|} d \mathbf{r}^{\prime} /
$$$$
\psi_{k}^{*}(\mathbf{r}) \psi_{k}(\mathbf{r}), \quad(2.16)
$$

${ }^{7}$ Subsequent to the original paper by Slater, there have been several attempts to add correlation corrections: S. Olszewski, Phys. Rev. 121, 42 (1961); J. E. Robinson, F. Bassani, B. S. Knox, and J. R. Schrieffer, Phys. Rev. Letters 9, 215 (1962); W. A. Harrison, Phys. Rev. 136, A1107 (1964); S. Lundqvist and C. W. Ufford, Phys. Rev. 139, A1 (1965). 
where the symbols $\mathbf{r}$ and $\mathbf{r}^{\prime}$ are understood to include electron spin coordinates and integration is understood to include summation over spin coordinates. One next assumes that the wave functions can be approximated by plane waves which results in

$$
v_{\mathbf{x} k}(\mathbf{r})=-\frac{k_{F}(\mathbf{r})}{\pi}\left[1+\frac{k_{F}^{2}(\mathbf{r})-k^{2}}{2 k k_{F}(\mathbf{r})} \ln \left|\frac{k+k_{F}(\mathbf{r})}{k-k_{F}(\mathbf{r})}\right|\right],
$$

where $k_{F}(\mathbf{r}) \equiv\left\{3 \pi^{2} n(\mathbf{r})\right\}^{1 / 3}$. Finally, one averages $v_{\mathbf{x} k}$ over the occupied state $k$, which results in

$$
v_{\mathbf{x}}(\mathbf{r})=-(3 / 2 \pi)\left\{3 \pi^{2} n(\mathbf{r})\right\}^{1 / 3} .
$$

In our procedure (neglecting correlation) we obtain, in place of Slater's $v_{\mathbf{x}}$

$$
\mu_{\mathbf{x}}(\mathbf{r})=-(1 / \pi)\left\{3 \pi^{2} n(\mathbf{r})\right\}^{1 / 3},
$$

smaller by a factor of $\frac{2}{3}$. From the discussion in Appendix I, it follows that while $\mu_{\mathbf{x}}$ gives the exchange correction of the density correct to order $|\nabla|^{2}$, inclusive, $v_{x}$ [as indeed any other function of $n(\mathbf{r})]$ leads to errors of order $|\nabla|^{2}$. The same comment applies to any extension of Slater's exchange to include correlation in the selfconsistent potential.

We may note that our result is equivalent to taking, not the average of (2.17), but rather its value at $k=k_{F}(\mathbf{r})$; i.e., the effective exchange potential for a state at the top of the Fermi distributions. This is physically understandable since density adjustments come about by redistribution of the electrons near the Fermi level.

(b) High density. This regime is characterized by the condition $r_{s} / a_{0} \ll 1$, where $a_{0}$ is the Bohr radius. In this case, the entire exchange and correlation energy is smaller than the kinetic energy by a factor of order $\left(r_{s} / a_{0}\right)$ and hence our inaccuracy in representing these portions becomes negligible.

The reader will have noticed that while in Eq. (2.3) we approximate the exchange and correlation energy by the expression valid for a slowly varying density, we made no approximation for the kinetic-energy functional $T_{s}[n]$ of Eq. (2.2). This procedure is responsible for the exactness of the high-density limit, even when the density is rapidly varying, such as in the vicinity of an atomic nucleus.

We now make a few further remarks about our approximation. If in Eq. (2.2), we had approximated $T_{s}[n]$ by its form appropriate to a system of slowly varying density,

$$
T_{s}[n] \rightarrow \int \frac{3}{10}\left(3 \pi^{2} n\right)^{2 / 3} n d \mathbf{r},
$$

we would have been led to the generalization of the Thomas-Fermi method suggested by Lewis. ${ }^{8}$ This method shares with the Thomas-Fermi method two shortcomings: (1) It leads to an infinite density near

\footnotetext{
${ }^{8}$ H. W. Lewis, Phys. Rev. 111, 1554 (1958).
}

an atomic nucleus, and (2) it does not lead to quantum density oscillations, ${ }^{4}$ such as the density fluctuations due to atomic shell structures. By not making the replacement (2.20), we avoid both of these shortcomings.

Let us now qualitatively discuss the appropriateness of our procedure for various classes of electronic systems.

In atoms and molecules one can distinguish three regions: (1) A region near the atomic nucleus, where the electronic density is high and therefore, in view of case $(b)$ above, we expect our procedure to be satisfactory. (2) The main "body" of the charge distribution where the electronic density $n(\mathbf{r})$ is relatively slowly varying, so that our approximation (2.3) for $\epsilon_{\mathrm{xe}}$ is expected to be satisfactory as discussed in case (a) above. (3) The "surface" of atoms and the overlap regions in molecules. Here our approximation (2.3) has no validity and therefore we expect this region to be the main source of error. We do not expect an accurate description of chemical binding. In large atoms, of course, this "surface" region becomes of less importance. (The surface is more satisfactorily handled in the nonlocal method described under $B$ below.)

For metals, alloys, and small-gap insulators we have, of course, no surface problem and we expect our approximation (2.3) to give a good representation of exchange and correlation effects. In large-gap insulators, however, the actual correlation energy will be considerably reduced compared to that of a homogeneous electron gas of the same density.

\section{B. Nonlocal Effective Potential}

Instead of the Hartree-type procedure discussed in Sec. IIA it is also possible to obtain a scheme which includes exchange effects exactly. We write in place of Eq. (2.3)

$$
E_{\mathrm{xc}}[n]=E_{\mathrm{x}}[n]+\int n(\mathbf{r}) \epsilon_{c}(n(\mathbf{r})) d \mathbf{r}
$$

where $E_{\mathrm{x}}[n]$ is the exchange energy of a Hartree-Fock system of density $n(\mathbf{r})$ and $\epsilon_{\mathrm{c}}(n)$ is the correlation energy per particle of a homogeneous electron gas. Applying this ansatz in conjunction with Eq. (2.2) and the stationary property of (2.1) leads to the following system of equations:

$$
\begin{aligned}
& \left\{-\frac{1}{2} \nabla^{2}+\varphi(\mathbf{r})+\mu_{\mathrm{c}}(\mathbf{r})\right\} \psi_{i}(\mathbf{r}) \\
& \qquad-\int \frac{n_{1}\left(\mathbf{r}, \mathbf{r}^{\prime}\right)}{\left|\mathbf{r}-\mathbf{r}^{\prime}\right|} \psi_{i}\left(\mathbf{r}^{\prime}\right) d \mathbf{r}^{\prime}=\epsilon_{i} \psi_{i}(\mathbf{r}), \\
& \text { where } \\
& \mu_{\mathrm{c}}=d\left(n \epsilon_{\mathrm{o}}\right) / d n, \\
& n_{1}\left(\mathbf{r}, \mathbf{r}^{\prime}\right)=\sum_{j=1}^{N} \psi_{j}(\mathbf{r}) \psi_{j}^{*}\left(\mathbf{r}^{\prime}\right)
\end{aligned}
$$

and $\varphi(\mathbf{r}), n(\mathbf{r})$ are defined as before, Eqs. (2.6) and (2.9). 
The energy is now

$$
\begin{aligned}
E=\sum_{1}^{N} \epsilon_{i}-\frac{1}{2} \int & \int \frac{n(\mathbf{r}) n\left(\mathbf{r}^{\prime}\right)}{\left|\mathbf{r}-\mathbf{r}^{\prime}\right|} d \mathbf{r} d \mathbf{r}^{\prime} \\
& +\frac{1}{2} \iint \frac{n_{1}\left(\mathbf{r}, \mathbf{r}^{\prime}\right) n_{1}\left(\mathbf{r}^{\prime}, \mathbf{r}\right)}{\left|\mathbf{r}-\mathbf{r}^{\prime}\right|} d \mathbf{r} d \mathbf{r}^{\prime} \\
& +\int n(\mathbf{r})\left\{\epsilon_{\mathrm{c}}(n(\mathbf{r}))-\mu_{\mathrm{c}}(n(\mathbf{r}))\right\} d \mathbf{r} .
\end{aligned}
$$

This procedure may be regarded as a Hartree-Fock method corrected for correlation effects. It is no more complicated than the uncorrected Hartree-Fock method but, because of the nonlocal operator appearing in $\mathrm{Eq}$. (2.22), very much more complicated than the method described in Sec. IIA. Since at least exchange effects are now treated exactly we must expect, in general, more accurate results than from the method of Sec. IIA. In particular, near the surface of an atom the effective potential now is correctly $(-1 / r)$ whereas in Sec. IIA it approaches zero much faster. Even here, however, correlation effects are not correctly described near the surface.

\section{FREE ENERGY; SPECIFIC HEAT}

We can generalize the consideration of the ground state to finite temperature ensembles by using the finite temperature generalization of Eq. (2.1) given by Mermin. ${ }^{9} \mathrm{He}$ has shown that the grand canonical potential can be written in the form

$$
\begin{aligned}
\Omega=\int v(\mathbf{r}) n(\mathbf{r}) d \mathbf{r}+\frac{1}{2} \int \frac{n(\mathbf{r}) n\left(\mathbf{r}^{\prime}\right)}{\left|\mathbf{r}-\mathbf{r}^{\prime}\right|} d \mathbf{r} d \mathbf{r}^{\prime} \\
\quad+G[n]-\mu \int n(\mathbf{r}) d \mathbf{r},
\end{aligned}
$$

where $G[n]$ is a unique functional of the density at a given temperature $\tau$ and $\mu$ is the chemical potential. For the correct $n$ this quantity is a minimum.

In analogy with (2.2) we now write

here

$$
G[n]=G_{s}[n]+F_{\mathrm{xc}}[n]
$$

$$
G_{s}[n] \equiv T_{s}[n]-\tau S_{s}[n],
$$

where $T_{s}[n]$ and $S_{s}[n]$ are, respectively, the kinetic energy and entropy of noninteracting electrons with density $n(\mathbf{r})$ at a temperature $\tau$; and $F_{\mathrm{xc}}[n]$ is, by definition, the exchange and correlation contribution to the free energy. For the latter quantity, we make the approximation

$$
F_{\mathrm{xc}}[n]=\int n(\mathbf{r}) f_{\mathrm{xc}}(n(\mathbf{r})) d \mathbf{r},
$$

where $f_{\mathbf{x c}}(n)$ is the exchange and correlation contribution to the free energy per electron of a uniform electron

\footnotetext{
${ }^{9}$ N. D. Mermin, Phys. Rev. 137, A1441 (1965).
}

gas of density $n$; i.e.,

$$
f_{\mathrm{xc}}(n) \equiv f(n)-f_{0}(n),
$$

where $f$ and $f_{0}$ are the free energies per electron of an interacting and noninteracting gas, respectively.

$$
0=\varphi(\mathbf{r})+\left(\delta G_{s}[n] / \delta n(\mathbf{r})\right)+\mu_{\mathrm{xc}}(n(\mathbf{r}))-\mu,
$$

where $\varphi(\mathbf{r})$ is given, as before, by Eq. (2.6) and

$$
\mu_{\mathrm{xc}}(n) \equiv d\left(n f_{\mathrm{xc}}(n)\right) / d n \text {. }
$$

Equation (3.6) is identical to the corresponding equation for a system of noninteracting electrons in the effective potential $\varphi+\mu_{\mathrm{xc}}$. Its solution is therefore determined by the following system of equations :

and

$$
\left\{-\frac{1}{2} \nabla^{2}+\varphi(\mathbf{r})+\mu_{\mathrm{xc}}(n(\mathbf{r}))\right\} \psi_{i}=\epsilon_{i} \psi_{i},
$$

$$
n(\mathbf{r})=\sum_{i=1}^{N}\left|\psi_{i}(\mathbf{r})\right|^{2} /\left\{e^{\left(\epsilon_{i}-\mu\right) / k \tau}+1\right\}
$$

$\mu$ is determined as usual by the total number of particles from Eq. (3.9). This value also represents our approximation for the chemical potential of the interacting system.

Of special interest for metals and alloys is the lowtemperature heat capacity. This may be obtained by making an expansion, in powers of $\tau$, of the above system of equations. An equivalent, but more convenient, method is as follows: From thermodynamics and Eq. (3.1) we have

$$
\begin{aligned}
& S[n] \equiv-\frac{\partial}{\partial \tau}(\Omega+\mu N)_{V}=-\int\left\{\varphi(\mathbf{r})+\frac{\delta G}{\delta n(\mathbf{r})}\right\} \\
& \times\left(\frac{\partial n(\mathbf{r})}{\partial \tau}\right)_{V} d \mathbf{r}-\left(\frac{\partial G[n]}{\partial \tau}\right)_{n(\mathrm{r}), V} .
\end{aligned}
$$

The integral vanishes because of the stationary property of $\Omega$, so that

$$
S[n]=-(\partial G[n] / \partial \tau)_{n(\mathrm{r}), V}
$$

The same argument, applied to a system of noninteracting electrons of density $n(\mathbf{r})$, gives

$$
S_{s}[n]=-\left(\partial G_{s}[n] / \partial \tau\right)_{n(\mathrm{r}), V} .
$$

Combining Eqs. (3.11), (3.12), (3.2), and (3.4), we obtain

$$
S[n]=S_{s}[n]+\int n(\mathbf{r})\left(\partial f_{\mathrm{xc}}(n) / \partial \tau\right)_{n(\mathrm{r}), v} d \mathbf{r} .
$$

For small $\tau$ it is well known that $S_{s}$ is given by

$$
S_{s}[n]=N \frac{1}{3} \pi^{2} k^{2} \tau g_{s}(\mu),
$$

where $g_{s}$ is the single-particle density of states in the effective potential $\varphi+\mu_{\mathbf{x c}}$ at zero temperature; further, $\left(\partial f_{\mathrm{xc}}(n) / \partial \tau\right)_{n(\mathrm{r}), V}=\frac{1}{3} \pi^{2} k^{2} \tau\left[g\left(\mu_{h}(n)\right)-g_{0}\left(\mu_{0}(n)\right)\right]$ 
where $\mu_{h}(n)$ and $\mu_{0}(n)$ are, respectively, the chemical potentials of an interacting and a noninteracting homogeneous gas of density $n$, and $g$ and $g_{0}$ are the respective densities of states. ${ }^{10}$

It follows immediately that the low-temperature heat capacity is given by

where

$$
C_{v}=\gamma \tau
$$

$\gamma=\frac{1}{3} \pi^{2} k^{2}\left[N g_{s}(\mu)+\int n(\mathbf{r})\left\{g\left(\mu_{h}(n)\right)-g_{0}\left(\mu_{0}(n)\right)\right\} d \mathbf{r}\right]$.

We shall not present a treatment, analogous to Sec. IIB, in which exchange effects are included exactly. The development is straightforward but leads to a wellknown divergence in the low-temperature specific heat.

\section{SPIN SUSCEPTIBILITY}

To obtain a theory of the spin susceptibility of an electron gas, we first extend the theory of HK to include the effects of spin interaction with an external magnetic field. The result is that if we take the field in the $z$ direction and write the magnetic-moment density as

$m(\mathbf{r})=-(1 / 2 c)\left\langle 0\left|\psi_{\uparrow} *(\mathbf{r}) \psi_{\uparrow}(\mathbf{r})-\psi_{\downarrow} *(\mathbf{r}) \psi_{\downarrow}(\mathbf{r})\right| 0\right\rangle$,

the ground-state energy can be written in the form

$$
\begin{aligned}
E_{v, H}=\int\{v(\mathbf{r}) n(\mathbf{r})-H(\mathbf{r}) m(\mathbf{r})\} d \mathbf{r} \\
+\frac{1}{2} \int \frac{n(\mathbf{r}) n\left(\mathbf{r}^{\prime}\right)}{\left|\mathbf{r}-\mathbf{r}^{\prime}\right|} d \mathbf{r} d \mathbf{r}^{\prime}+G[n(\mathbf{r}), m(\mathbf{r})],
\end{aligned}
$$

where $G$ is a universal functional of $n$ and $m$, and the correct $m(\mathbf{r}), n(\mathbf{r})$ make (4.2) a minimum.

For small $m$ we expand $G$ in the form

$$
G=G[n]+\frac{1}{2} \int G\left(\mathbf{r}, \mathbf{r}^{\prime} ;[n]\right) m(\mathbf{r}) m\left(\mathbf{r}^{\prime}\right) d \mathbf{r} d \mathbf{r}^{\prime}+\cdots ;
$$

the linear term vanishes for a paramagnetic system in which $m \equiv 0$ when $H \equiv 0$. From the stationary property of (4.2) we find, for small $H$, that $n$ is unchanged to first order and that

$$
-H(\mathbf{r})+\int G\left(\mathbf{r}, \mathbf{r}^{\prime} ;[n]\right) m\left(\mathbf{r}^{\prime}\right) d \mathbf{r}^{\prime}=0,
$$

where $n$ is the zero-field density. We now formally invert this equation, which gives

$$
m(\mathbf{r})=\int G^{-1}\left(\mathbf{r}, \mathbf{r}^{\prime} ;[n]\right) H\left(\mathbf{r}^{\prime}\right) d \mathbf{r}^{\prime}
$$

For a uniform field this gives for the susceptibility

$$
\chi[n]=\frac{1}{V} \frac{\partial}{\partial H} \int m(\mathbf{r}) d \mathbf{r}=\int G^{-1}\left(\mathbf{r}, \mathbf{r}^{\prime} ;[n]\right) d \mathbf{r} d \mathbf{r}^{\prime} .
$$

${ }^{10}$ J. M. Luttinger, Phys. Rev. 119, 1153 (1960).
So far everything is formal and exact. We now write, in the spirit of the previous sections,

$G^{-1}\left(\mathbf{r}, \mathbf{r}^{\prime} ;[n]\right) \equiv G_{s}^{-1}\left(\mathbf{r}, \mathbf{r}^{\prime} ;[n]\right)+G_{\mathbf{x c}}^{-1}\left(\mathbf{r}, \mathbf{r}^{\prime} ;[n]\right)$.

The second term we approximate as for a slowly varying gas, which gives

$$
\chi[n]=\chi_{s}[n]+\frac{1}{V} \int\left[\chi(n(\mathbf{r}))-\chi_{0}(n(\mathbf{r}))\right] d \mathbf{r},
$$

where

$$
\chi_{s}[n]=(1 / 2 c)^{2}(N / V) \times g_{s}(\mu),
$$

and $\chi(n), \chi_{0}(n)$ are, respectively, the susceptibilities for uniform systems with and without interactions.

\section{APPENDIX I: GRADIENT EXPANSION OF THE DENSITY}

In this Appendix we show that for a system of slowly varying density our procedure gives the density correct to order $|\nabla|^{2}$ inclusive. When dealing with such a system we may proceed in two entirely equivalent ways: (1) We can solve the self-consistent equations, Eqs. (2.8) and (2.9), for $n(\mathbf{r})$, and (2) we can go back to the underlying variational principle (2.5), make a gradient expansion and determine $n(\mathbf{r})$ directly. We shall here follow the second route to estimate the errors in $n(\mathbf{r})$.

From (2.5) and the expansion (2.12) of $T_{s}[n]$, we obtain

$\mu=\varphi(\mathbf{r})+\mu_{h}(n)-t^{(2) \prime}(n)|\nabla n|^{2}-2 t^{(2)}(n) \nabla^{2} n+O\left(\nabla^{4}\right)$,

where $\mu$ is the chemical potential [cf. HK, Eq. (68)]. Note however that because of our approximation of keeping only the first term in (2.11), some other contributions of order $|\nabla|^{2}$ are missing in (A1.1).

To solve (A1.1), let us write the external charge density as

$$
n_{\mathrm{ext}}(\mathbf{r}) \equiv f_{0}\left(\mathbf{r} / r_{0}\right)
$$

where $r_{0} \rightarrow \infty$ (slow spatial variation), and try the ansatz

where

$$
n(\mathbf{r})=n_{0}(\mathbf{r})+n_{1}(\mathbf{r})
$$

$$
n_{0}(\mathbf{r})=f_{0}\left(\mathbf{r} / r_{0}\right)
$$

exactly neutralizes the external charge and $n_{1}$ is assumed to approach zero as $\boldsymbol{r}_{0} \rightarrow \infty$. Neglecting, for the moment, the terms of order $|\nabla|^{2}$ in (A1.1) and substituting (A1.3) into (A1.1), we obtain

$\mu=\int \frac{n_{1}\left(\mathbf{r}^{\prime}\right)}{\left|\mathbf{r}-\mathbf{r}^{\prime}\right|} d \mathbf{r}^{\prime}+\mu_{h}\left(n_{0}\right)+n_{1}(\mathbf{r}) \mu_{h}^{\prime}\left(n_{0}\right)+O\left(n_{1}^{2}\right)$.

Now define

and write

$$
\mathbf{R} \equiv \mathbf{r} / \boldsymbol{r}_{0}
$$

$$
n_{1}(\mathbf{r}) \equiv f_{1}(\mathbf{R})
$$


With this notation, (A1.5) becomes

$$
\begin{aligned}
\mu=r_{0}^{2} \int \frac{f_{1}\left(\mathbf{R}^{\prime}\right)}{\left|\mathbf{R}-\mathbf{R}^{\prime}\right|} d \mathbf{R}^{\prime}+\mu_{h}\left(f_{0}(\mathbf{R})\right) \\
\\
+f_{1}(\mathbf{R}) \mu_{h}{ }^{\prime}\left(f_{0}(\mathbf{R})\right)+O\left(f_{1}^{2}\right) .
\end{aligned}
$$

We may now write

$$
f_{1}(\mathbf{R})=\left(1 / r_{0}^{2}\right) f_{1}^{(2)}(\mathbf{R})+\left(1 / r_{0}^{4}\right) f_{1}{ }^{(4)}(\mathbf{R})+\cdots,
$$

and

$$
\mu=\mu^{(0)}+\left(1 / r_{0}^{2}\right) \mu^{(2)}+\cdots .
$$

The first term of Eq. (A1.9) is correctly determined by Eq. (A1.8) and not affected either by the inclusion of terms of order $\nabla^{2}$ in (A1.5) or by the terms of order $f_{1}{ }^{2}$ in (A1.8). Hence, in spite of the errors of order $\nabla^{2}$ in (A1.1), the density given by our procedure is correct to order $1 / r_{0}^{2}$ or $|\nabla|^{2}$, inclusive. Equation (A1.8) shows that this curious result stems from the infinite range of the Coulomb interaction.

\section{APPENDIX II : EFFECT OF RAPID DENSITY OSCILLATION ON EXCHANGE AND CORRELATION}

In Eq. (2.3), we approximated $E_{\mathrm{xc}}[n]$ by the first term in the gradient expansion. In actual physical systems, there are quantum density oscillations ${ }^{4}$ whose effects on exchange and correlation are not included in the approximation (2.3). Now we put forward a correction to (2.3) to include such effects.

In HK, the gradient expression for the energy functional is partially summed such that it is also correct for a system of almost constant density ${ }^{1}$ even when the density fluctuations are of short wavelength ${ }^{11}$ :

$$
\begin{aligned}
G[n]=\int g_{0}(n(\mathbf{r})) d \mathbf{r}-\frac{1}{2} \int K\left(\mathbf{r}-\mathbf{r}^{\prime} ; n(\overline{\mathbf{r}})\right) \\
\times\left\{n(\mathbf{r})-n\left(\mathbf{r}^{\prime}\right)\right\}^{2} d \mathbf{r} d \mathbf{r}^{\prime},
\end{aligned}
$$

where $K\left(\mathbf{r}-\mathbf{r}^{\prime} ; n\right)$ is determined by the polarizability of a homogeneous electron gas at density $n$, and $\overline{\mathbf{r}}=\frac{1}{2}\left(\mathbf{r}+\mathbf{r}^{\prime}\right)$. To the same approximation,

$$
\begin{aligned}
E_{\mathrm{xc}}[n]=\int n(\mathbf{r}) \epsilon_{\mathrm{xc}}(n(\mathbf{r})) & d \mathbf{r}-\frac{1}{2} \int K_{\mathrm{xc}}\left(\mathbf{r}-\mathbf{r}^{\prime} ; n(\overline{\mathbf{r}})\right) \\
& \times\left\{n(\mathbf{r})-n\left(\mathbf{r}^{\prime}\right)\right\}^{2} d \mathbf{r} d \mathbf{r}^{\prime}
\end{aligned}
$$

where $K_{\mathbf{x e}}$ is the difference between $K$ of the interacting homogeneous gas and that of the noninteracting gas at the same density. We believe that for an infinite system,

11 The second term of HK, Eq. (83) is in error; it should be

$$
-\frac{1}{2} \int K\left(\mathbf{r}^{\prime} ; n(\mathbf{r})\right)\left\{n\left(\mathbf{r}+\frac{1}{2} \mathbf{r}^{\prime}\right)-n\left(\mathbf{r}-\frac{1}{2} \mathbf{r}^{\prime}\right)\right\}^{2} d \mathbf{r}^{\prime} .
$$

The kernel $K$ has the same meaning as in HK. such as a metal or an alloy, the second term on the right-hand side of (A2.2) accounts adequately for the effect of rapid density change on exchange and correlation.

This $E_{\mathbf{x c}}[n]$ again leads to a set of Hartree-type equations like Eq. (2.8), with an addition to the effective potential given by

$$
\begin{aligned}
& -\frac{1}{2} \int\left\{\partial K_{\mathbf{x c}}\left(\mathbf{r}^{\prime} ; n(\mathbf{r})\right) / \partial n(\mathbf{r})\right\} \\
& \quad \times\left\{n\left(\mathbf{r}+\frac{1}{2} \mathbf{r}^{\prime}\right)-n\left(\mathbf{r}-\frac{1}{2} \mathbf{r}^{\prime}\right)\right\}^{2} d \mathbf{r}^{\prime} \\
& \quad-2 \int K_{\mathrm{xc}}\left(\mathbf{r}-\mathbf{r}^{\prime} ; n(\overline{\mathbf{r}})\right)\left\{n(\mathbf{r})-n\left(\mathbf{r}^{\prime}\right)\right\} d \mathbf{r}^{\prime} .
\end{aligned}
$$

Note that in the random-phase approximation $K_{\mathbf{x c}}$ vanishes. Hence, in a calculation which includes the effective potential (A2.3), we need reliable estimates of $K_{\text {xc }}$, calculated beyond the random-phase approximation, which are not available at present.

The addition of (A2.3) to the effective potential obviously makes the solution of the self-consistent equations much more difficult. However, assuming that the modification of $n(\mathbf{r})$ produced by this term is small, one may calculate $n(\mathbf{r})$ and $E$ first without including it, and then, because of the stationary property, Eq. (2.5), one can obtain the correction to the energy by evaluating the second term in (A2.2) with the unmodified density.

Note added in proof. We should like to point out that it is possible, formally, to replace the many-electron problem by an exactly equivalent set of self-consistent one-electron equations. This is accomplished quite simply by using the expression (2.2) [without the approximation (2.3)] in the energy variational principle. This leads to a set of equations, analogous to Eqs. (2.4)-(2.9), but with $\mu_{\mathrm{xo}}(n)$ replaced by an effective one-particle potential $v_{\mathbf{x}}$, defined formally as

$$
v_{\mathrm{xc}}(\mathbf{r}) \equiv \delta E_{\mathrm{xc}}[n] / \delta n(\mathbf{r}) .
$$

Of course, an explicit form of $v_{\mathbf{x o}}$ can be obtained only if the functional $E_{\mathrm{xc}}[n]$, which includes all many-body effects, is known. This effective potential will reproduce the exact density and the exact total energy is then given by

$$
\begin{aligned}
E=\sum_{1}^{N} \epsilon_{i}-\frac{1}{2} \iint \frac{n(\mathbf{r}) n\left(\mathbf{r}^{\prime}\right)}{\left|\mathbf{r}-\mathbf{r}^{\prime}\right|} d \mathbf{r} d \mathbf{r}^{\prime}+E_{\mathbf{x c}}[n] & \\
& -\int v_{\mathrm{xo}}(\mathbf{r}) n(\mathbf{r}) d \mathbf{r} .
\end{aligned}
$$

Of course, if we make the approximation (2.3) for $E_{\mathbf{x c}}$ the above exact formulation reverts to the approximate theory of Sec. II. 